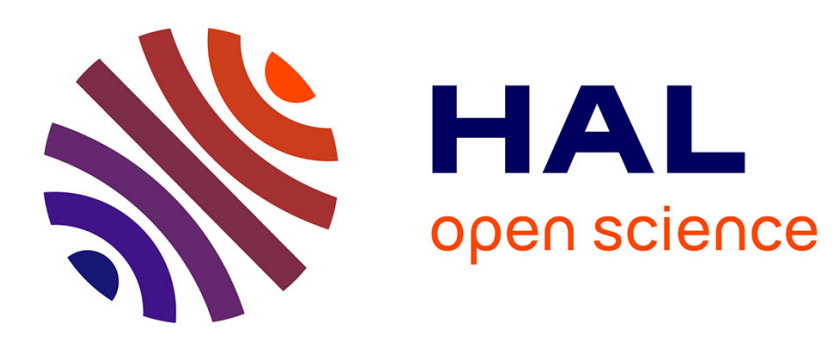

\title{
Two Levels Modeling for the Optimization of Electromagnetic Actuators
}

\author{
Benoît Delinchant, G. Gruosso, Frédéric Wurtz
}

\section{To cite this version:}

Benoît Delinchant, G. Gruosso, Frédéric Wurtz. Two Levels Modeling for the Optimization of Electromagnetic Actuators. IEEE Transactions on Magnetics, 2009, 45 (3), pp.1724-1727. hal-00384151

\section{HAL Id: hal-00384151 \\ https://hal.science/hal-00384151}

Submitted on 14 May 2009

HAL is a multi-disciplinary open access archive for the deposit and dissemination of scientific research documents, whether they are published or not. The documents may come from teaching and research institutions in France or abroad, or from public or private research centers.
L'archive ouverte pluridisciplinaire HAL, est destinée au dépôt et à la diffusion de documents scientifiques de niveau recherche, publiés ou non, émanant des établissements d'enseignement et de recherche français ou étrangers, des laboratoires publics ou privés. 


\title{
Two levels modeling for the optimization of electromagnetic actuators
}

\author{
B. Delinchant ${ }^{(+)}$, G. Gruosso ${ }^{(*)}$ (Member IEEE), F. Wurtz ${ }^{(+)}$(Member IEEE) \\ ${ }^{(+)}$G2ELab - Grenoble Electrical Engineering Lab, ENSE3, 38402 Grenoble, FRANCE \\ ${ }^{(*)}$ Politecnico di Milano- Dipartimento di Elettronica e Informazione, Piazza Leonardo da Vinci, 32-20133 Milano, Italy \\ Benoit.Delinchant@inpg.fr - gruosso@elet.polimi.it
}

\begin{abstract}
In this paper a two stage modelling, to ensure a good and fast optimization procedure for microdevices, is be presented. This methodology is supported by three non-commercial software. The first one is dedicated to analytical modelling of non ferromagnetic electromagnetic devices, the second one is dedicated to numerical modelling of electromagnetic devices, and the last is dedicated to constrained based optimization. Issues and solutions of such a methodology are highlighted, and illustrated by designing a matrix of actuators for an adaptive optic.
\end{abstract}

\section{INTRODUCTION AND METHODOLOGY}

Adaptive optic is based on a deformable reflector actuated by a matrix of electromagnetic actuators [1]. Each actuator is commanded separately and moves in real time the reflecting surface to balance atmosphere turbulence and avoid image deformations.
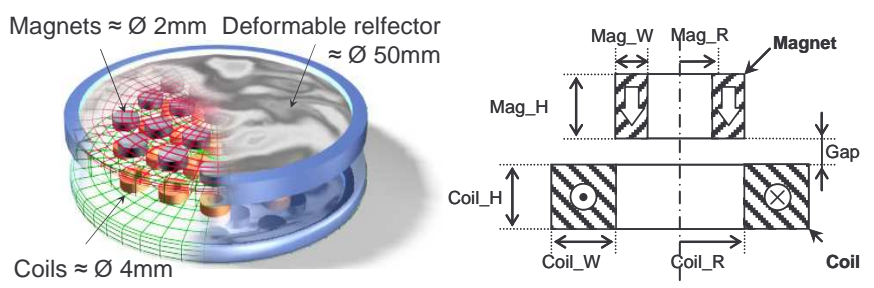

Fig. 1. Optical system and actuator creating mirror micro displacement

Force of the initial device is $26 \mathrm{mN}$ at Gap $=0.5 \mathrm{~mm}$, the mass is equal to $18 \mathrm{mg}$. The aim of the design optimization is to improve actuators regarding actuating force (force at $\mathrm{Gap}=0.5 \mathrm{~mm}$ must be $30 \mathrm{mN}$ ) and magnet mass minimization.

To perform such a design, optimization procedure is required. Several strategies are available; the simplest is to optimize with direct calls to the numerical simulation solver, but it is a very expensive in terms of computational efforts; an other is to create a response surface from a numerical solver which is then optimized [2]. A third solution has been chosen for this study, which is also based on two level modeling and which can be seen as a manual manifold mapping technique [3] including knowledge of the designer.

First modeling level is based on an analytical description of the device to perform the optimization, and then refining the solution with a second modeling level using a numerical solver that takes into account phenomena like magnetic demagnetization.

\section{MODELING FORMULATIONS}

\section{A. Analytical formulation}

Our analytical modeling is based on integral formulations which are symbolically solved for basic shapes [4]. Other symbolic solutions, based on elliptic integrals can also be used
[5]. Magnetic field produced by magnets is computed using Coulombian equivalent charge method; Biot and Savart law is applied for current sources. Forces and torques acting on magnets are computed using an equivalent surface charges method like electrostatic devices; Laplace law is used on conductors. Based on a magnetic components library and the application of superposition theorem, software has been developed to produce automatically this kind of analytical model from a geometrical and physical description [6].

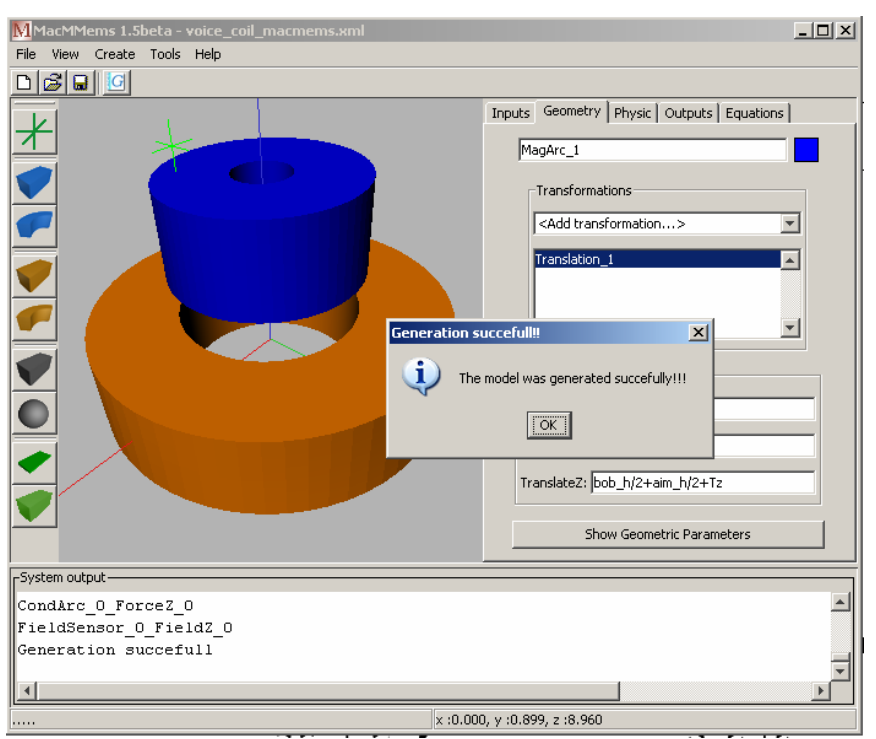

Fig. 2. Software generating an analytical model from a parameterized geometry and physic [6].

On the one hand, computation time of this kind of model is very short, which is important for an optimization process. Moreover, symbolic gradients are available, allowing very constrained design. On the other hand, this modeling lacks of accuracy. For example, magnet magnetization is homogenous and constant.

\section{B. Numerical Method: Mixed FFEF-BEM Modeling}

Our numerical solver is based on a Finite Formulation of Electromagnetic Fields (FFEF), coupled with integral boundary conditions based on magnetization integrals [7].

The method is based on the use of scalar global variables, obtained by integrating field variables on a double system of meshes, strictly connected by relations of duality. Global variables are distinguished in: Configuration Variables $(\mathrm{CV})$ associated to the primal mesh and Source Variables (SV), associated to the dual one. CV involved in the actual study are magnetic $\varphi$ fluxes on primal faces and circulation $p$, line integral of magnetic vector potential on primal edges. While $\mathrm{SV}$ are magnetomotive forces $F(\mathrm{mmf})$ on dual edges and $i$ 
current on dual faces. According with [7] the topological equations are the following :

$$
\begin{aligned}
& {[C]\{p\}=\{\varphi\}} \\
& {[\tilde{C}]\{F\}=\{i\}}
\end{aligned}
$$

being $[C]$ and $[\tilde{C}]$ curl matrices on primal and dual complex, respectively. The constitutive equation is:

$$
[F]=[R]_{V}\{\varphi\}-\left\{F_{M}\right\}
$$

where $[R]_{V}$ is the reluctance matrix, that takes into account the magnetic anisotropy, and $F_{M}$ is the mmf due to magnetization sources on dual edges.

The solution of the previous equations requires a proper set of boundary conditions. This fact requires the discretization of the air surrounding the analyzed device. In order to limit the discretization of the problem to its ferromagnetic part only, the presented hybrid formulation adds some integral terms to the equations for the relations of the primal edges lying on the boundary of the ferromagnetic part. Thus inner edges and boundary ones have to be treated in different way. For each inner edge an equation can be written by imposing the satisfaction of Ampere law on the contour of the dual face associated to it. For outer edges it is not possible to write an equation of the same type, because there is no associated dual face contained as whole inside the domain. Thus exterior part of the integration path can be efficiently treated by a magnetic scalar potential difference as:

$\int_{a}^{b} H \cdot d l=\psi(a)-\psi(b)$

where $a$ and $b$ are the points where the dual face contour intersects the mesh boundary.

An integral equation can be written for the external part of the domain as:

$$
\alpha \psi(\mathbf{r})-\int_{S} \psi\left(\mathbf{r}^{\prime}\right) \frac{\partial G\left(\mathbf{r}, \mathbf{r}^{\prime}\right)}{\partial \mathbf{n}} d S+\int_{S} \frac{\partial \psi\left(\mathbf{r}^{\prime}\right)}{\partial \mathbf{n}} G\left(\mathbf{r}, \mathbf{r}^{\prime}\right) d S=0
$$

Where is $\alpha$ constant which is equal to $\pi$ if point $\mathbf{r}$ lies on a plane surface and $G\left(\mathbf{r}, \mathbf{r}^{\prime}\right)$ is the free space Green function.

By starting from this formulation the force calculation has been performed by means of Maxwell stress tensor. This method is usually considered critic, in particular, the value coming out of the integration of Maxwell tensor can be affected by a large error when the integration surface crosses the finite element mesh due to local inaccuracies of computed values of magnetic flux density. As it was pointed out in [8], this problem can be overcome by the use of hybrid techniques where magnetic field is obtained by integral formulas whose accuracy is largely independent of the mesh structure.

This modeling has been used to overcome some hypothesis of the previous one, including for example the demagnetization of the hard magnetic material.

\section{OPTIMIZATION STRATEGY}

\section{A. Simple optimization on the analytical model}

Optimization over the analytical model, to achieve design requirements, does not work without some safety measures.
Indeed, it will be shown that optimizer goes, most of time, out of the model validity domain. It is possible to constrain parameters to stay in a restricted area, but these constraints are artificial and then optimal solution too.

At first stage an optimisation with a constraint limiting the solution to the range of validity has be done in the CADES framework [9]. The parameter meaning and their variation range is reported in Tab.I. The objective of the optimization are:

- Volumic force optimization (to improve reactivity during regulation of micro mirrors)

- Nominal force is constrained to be equal to $0.03 \mathrm{~N}$

- Magnet aspect ratio in constrained to be $<1$ (magnet high $>$ magnet width)

TABLE I

PARAMETER VALUE AND RANGE OF VARIATION

\begin{tabular}{l|llll}
\hline Parameter & Description & Unit & Value & Type \\
\hline Coil_W & Coil Width & $\mathrm{mm}$ & 1 & fixe \\
Coil_H & Coil High & $\mathrm{mm}$ & 1 & fixe \\
Coil_R & Coil Radius & $\mathrm{mm}$ & 1 & fixe \\
J & Current density & $\mathrm{A} / \mathrm{mm}^{2}$ & 100 & fixe \\
Mz & Magnetization & $\mathrm{T}$ & 1 & fixe \\
Gap & Distance & $\mathrm{Mm}$ & 0.5 & fixe \\
Mag_W & Magnet Width & $\mathrm{mm}$ & 0.5 & Optimizable $[0.1 ; 2]$ \\
Mag_H & Magnet High & $\mathrm{mm}$ & 1 & Optimizable $[0.1 ; 2]$ \\
Mag_R & Magnet Radius & $\mathrm{mm}$ & 0.5 & Optimizable $[0 ; 2]$ \\
\hline
\end{tabular}

In Fig. 3 the configuration before and after the optimization is reported. The objective is obtained reaching the good force value but the maximal value of the aspect ratio, creating an artificial optimal solution (fig. 4).
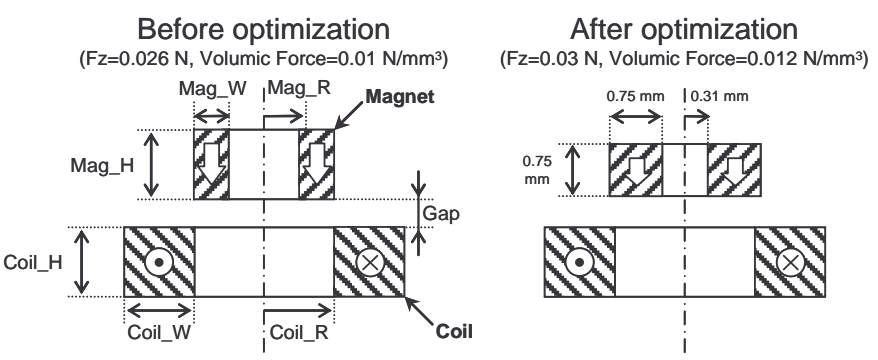

Fig. 3. Configuration before and after constrained optimization
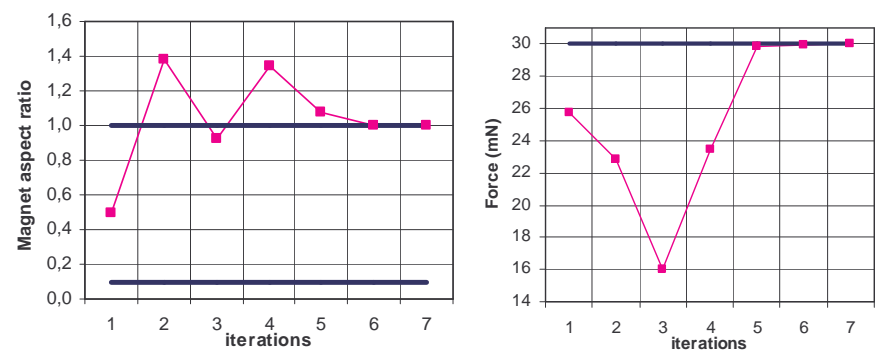

Fig. 4. Converge of constraints during optimization (force $=30 \mathrm{mN}$ and magnet aspect ration $=1$ )

It is possible to see, from the numerical simulation, as the demagnetization of the magnet takes a significant role in the determination of the force (fig.5). Under these hypotheses the value of the calculate force is $27.8 \mathrm{mN}$ different from the desidered value of $30 \mathrm{mN}$. 


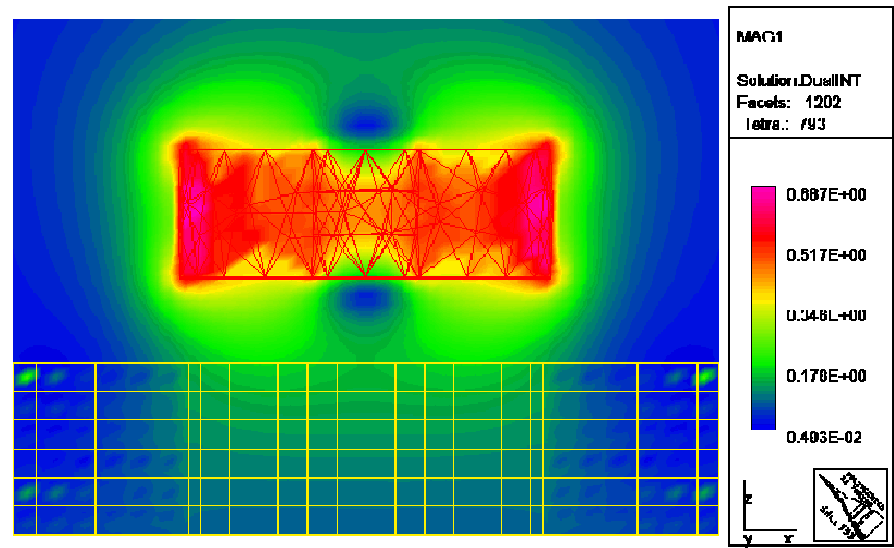

Fig. 5. Effect of demagnetization simulated using numerical method (Tesla)

\section{B. Optimization without validity limitation}

In order to avoid the artificial solution obtained in the previous case, the solution is to try to relax constraints to see where the algorithm converge and if it is far from our artificial limit of the form factor $=1$. At this stage the objectives and constraints are:

- Volumic force optimization (to improve reactivity during regulation of micro mirrors)

- Nominal force is constrained to be equal to $0.03 \mathrm{~N}$

- Magnet aspect ratio is free

After optimization $\left(\mathrm{Fz}=0.03 \mathrm{~N}\right.$, Volumic Force $\left.=13.6 \mathrm{mN} / \mathrm{mm}^{3}\right)$

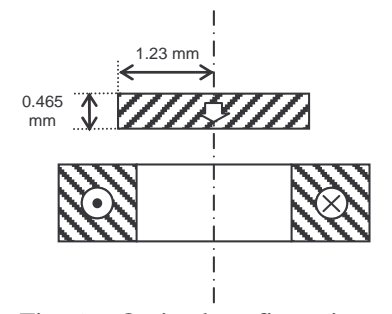

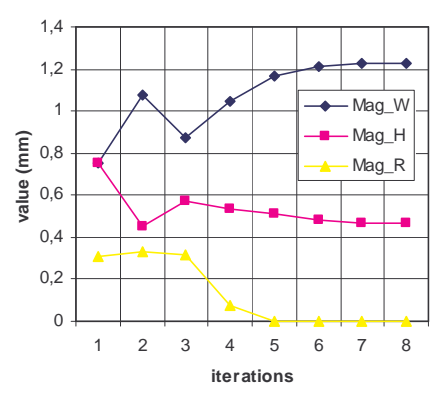

In fig. 6 the optimal configuration is shown together with the parameter variation versus iteration number.

We can see that magnet form factor (aspect ratio) converge to an optimal value of 2.64 (fig. 7), moreover, there is no hole inside the magnet leading to a real form factor of $2.65 \times 2$ $=5.3$ which is probably too big regarding demagnetization effect.
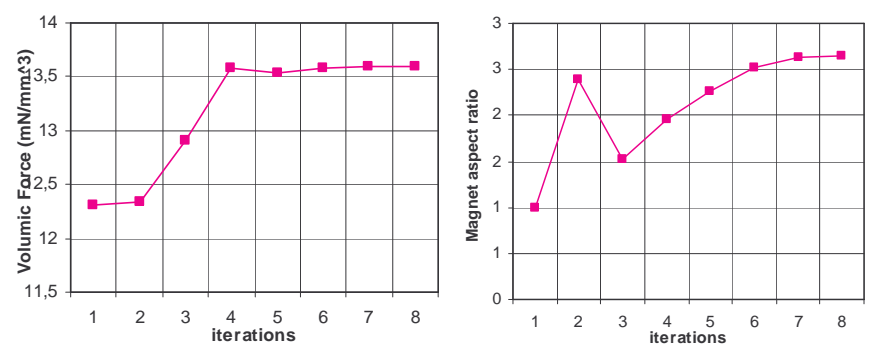

Fig. 7. Volumic force and aspect ratio versus iteration number

We have to check if our modeling is good in such a configuration thanks to an other modeling techniques including demagnetization curve of the magnet. The computed force in this condition is equal to $27 \mathrm{mN}$. This is the proof that our optimization is false due to the magnetization effect that is neglected in the analytical model (fig. 8).

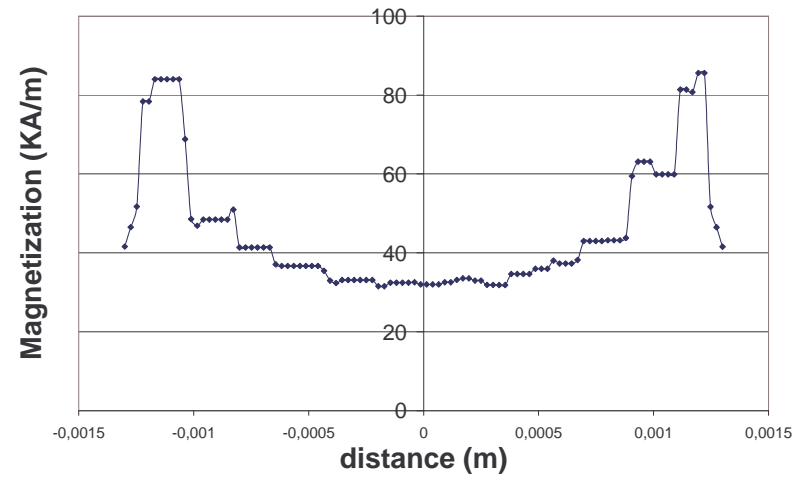

Fig. 8. Demagnetization versus radius in the optimized configuration

\section{A Strategy to overcome such artificial constraints}

How to use our optimization model knowing that the optimal solution is out of the range of validity?

A good solution is to use manifold mapping technique but it requires to make both models computable automatically, and implementing the mathematical algorithm in a supervisor, which is not currently our case.

A quick solution is then proposed including the knowledge of the designer on the domain restriction of its modeling. The solution consists of plotting a curve of optimal configurations for several value of a parameter representing the modeling hypothesis rightness (in our case : magnet aspect ration)

The technique used to plot such a curve is based on a set of optimizations where Magnet aspect ration is constrained to a fixed value from 1 to 3 by steps of 0.25 ( 9 automated sequential optimizations). It is automated thanks to the CADES optimization framework [9]. It takes 40 iterations (20 minutes). For each optimal configuration, a simulation is run to check rightness.
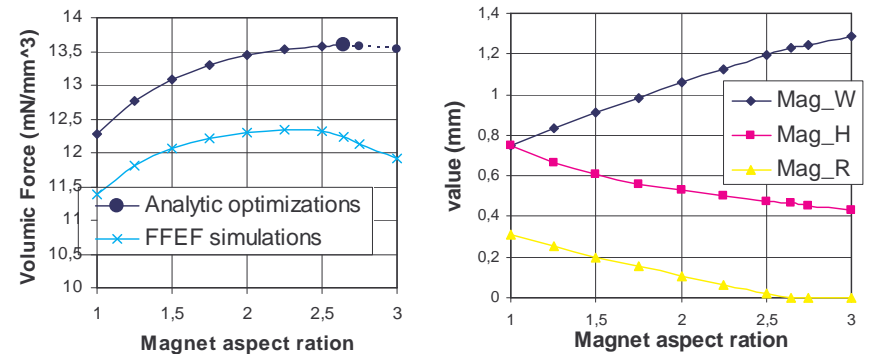

Fig. 9. Set of optimal design versus magnet aspect ratio.

The numerical modeling curve shows a displacement of the optimal point to better aspect ratio giving a good optimal solution regarding demagnetization effect. Indeed, even if demagnetization has an effect, the structure of micro-actuators gives an optimal solution with an aspect ratio of about 2.25.

We can notice that optimal solution of part B (aspect ratio = 2.64) is defined when magnet radius (Mag_R) reach zero.

As it can be seen on fig. 10 that real force decrease when analytical model trusts on a constant value of $30 \mathrm{mN}$. This decrease is a proof that magnet aspect ratio is a good criteria for the estimation of rightness of the analytical model. 


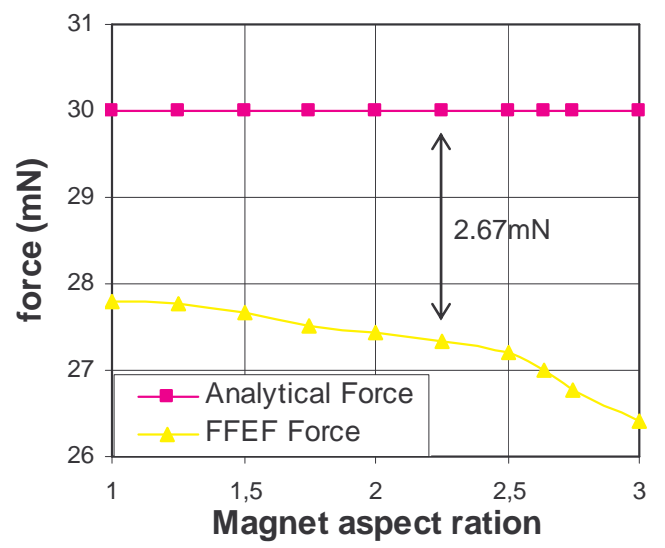

Fig. 10. Force for the set of optimal configuration.

This figure shows also a difference of $2.67 \mathrm{mN}$ between analytical and numerical model at the optimal magnet aspect ration founded before. This difference has to be cancelled to reach the real design specifications.

A final optimization is then performed to adjust the force with a kind of manual manifold mapping technique. This optimization tries to reach a force constrained to a compensated value of $30+2.67 \mathrm{mN}$.

Only 4 iterations are required to converge to the desired force, giving the final optimal configuration in fig 11 . This solution has been simulated with the fine model, computing a force of $30 \mathrm{mN}$ with a maximal volumic force of 12.1 $\mathrm{mN} / \mathrm{mm}^{3}$.
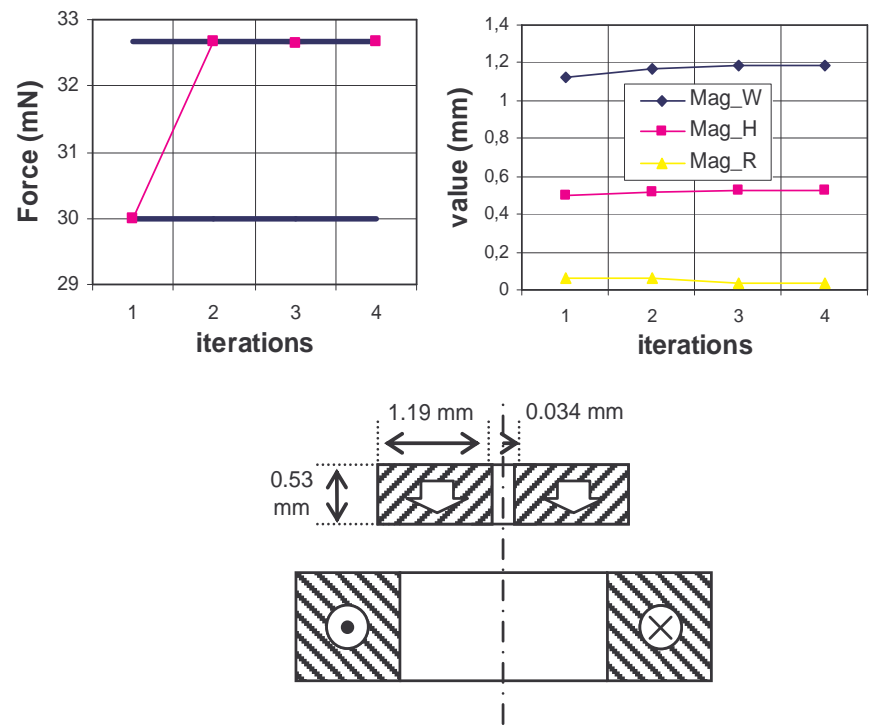

Fig. 11. Convergence to the optimal configuration

\section{CONCLUSIONS}

In this study, a methodology using two level modeling and optimization tool was demonstrated on an simple application. This methodology can be applied on more complex systems, even with several axis of hypothesis where curve as to be changed by $n$-dimensional plots.

It is based on knowledge about electromagnetic hypothesis of modeling. Indeed, design has to find the parameter which characterize the modeling hypothesis of coarse model (analytic one here)

Such strategies need fast modeling (coarse model), coupled with optimization algorithm and an accurate model (simulation tool) to check some specific configurations.

\section{V.REFERENCES}

[1] O. Cugat, S. Basrour, C. Divoux, P. Mounaix and G. Reyne "Deformable magnetic mirror for adaptive optics: technological aspects", Sensors and Actuators A: Physical, Volume 89, Issues 1-2, 20 March 2001, Pages 1-9

[2] K. N'Guessan ,V. Leconte, J.L. Coulomb, F. Wurtz, V. Mazauric, "Comparison of two optimization strategies for the design of an ultrafast actuator", OIPE, Grenoble, FRANCE, 6-8 Sept. 2004

[3] Echeverria, D. and Hemker, P.W. (2006), "On the Manifold-Mapping Optimization Technique”, Technical Report MAS-R0612, Centrum voor Wiskunde en Informatica (CWI), Amsterdam, June.

[4] Rakotoarison, H. L.; Yonnet, J.-P.; Delinchant, B. "Using Coulombian Approach for Modeling Scalar Potential and Magnetic Field of a Permanent Magnet With Radial Polarization", IEEE Transactions on Magnetics, vol. 43, n 4, pp. 1261-1264, Apr. 2007

[5] Ravaud,R.,G. Lemarquand,V. Lemarquand,and C. Depollier, "Analytical calculation of the magnetic field created by permanentmagnet rings," IEEE Trans. Magn.,V ol. 44,No. 8, 1982-1989,Aug. 2008 .

[6] Rakotoarison, H.L. Delinchant, B. Cugat, O., "Methodology and tool for generating semi-analytical models Used to pre-design electromagnetic MEMS (Mag-MEMS)", CEFC 2006, USA, Mai 2006, p444, ISBN: 1-4244-0320-0

[7] G. Giuffrida, C. Gruosso and M. Repetto, "Finite Formulation of nonlinear magneto-statics with integral boundary conditions," IEEE Transactions on Magnetics, vol. 42, n ${ }^{\circ}$ 5, May, 2006, p 1503-1511

[8] Z. Ren and A. Razek, "Force calulation by Maxwell stress tensor in 3D hybrid finite element - boundary element integral formulation", IEEE Transactions on Magnetics, vol. 26, pp. 2774-2776, 1990.

[9] B. Delinchant, D. Duret, L. Estrabaut, L. Gerbaud, H. Nguyen Huu, B. Du Peloux, H.L. Rakotoarison, F. Verdiere, F. Wurtz "An optimizer using the software component paradigm for the optimization of engineering systems", COMPEL 2007, Vol. 26, Issue 2, Page: 368 - 379 